BIO Web of Conferences 2, 03005 (2014)

DOI: $10.1051 /$ bioconf/ 20140203005

C) Owned by the authors, published by EDP Sciences, 2014

\title{
Clathrate hydrate FTIR spectroscopy - infrared signatures, astrophysical significance
}

\author{
E. Dartois ${ }^{1}$, M. Bouzit ${ }^{1}$, Ph. Duret ${ }^{1}$, and B. Schmitt ${ }^{2}$ \\ ${ }^{1}$ Institut d'Astrophysique Spatiale, UMR 8617, Université Paris-Sud, bât. 121, 91405 Orsay, France \\ ${ }^{2}$ Institut de Planétologie et d'Astrophysique de Grenoble, UMR 5274, Bâtiment D de Physique, BP \\ 53, 38041 Grenoble Cedex 9, France
}

\begin{abstract}
Clathrate hydrates, ice inclusion compounds, are of major importance for the Earth's permafrost regions and may control the stability of gases in many astrophysical bodies such as the planets, comets and possibly interstellar grains. Their physical behavior may provide a trapping mechanism to modify the absolute and relative composition of icy bodies that could be the source of late-time injection of gaseous species in planetary atmospheres or hot cores. In this study, we provide and discuss laboratory-recorded infrared signatures of clathrate hydrates in the near to mid-infrared and the implications for space-based astrophysical tele-detection in order to constrain their possible presence.
\end{abstract}

\section{Method}

A gold coated copper cell is thermally coupled to a liquid He-transfer cold finger, placed in a highvacuum, evacuated cryostat $\left(\mathrm{P}<10^{-7} \mathrm{mbar}\right) .2 \mathrm{~mm}$ thick infrared transmitting zinc selenide windows are sealed with indium gaskets, and allow the spectrometer beam to record the clathrate hydrate spectra. A soldered stainless steel injection tube, brazed to the lower part of the cell allows for the entrance of gas or its evacuation. Dartois et al. (2010). To prepare the clathrate, vapor water is injected in the evacuated cell precooled to $240 \mathrm{~K}$, and condenses on the windows. The guest gases are injected, with a predefined molar abundance ratio, in large pressure excess. The cell is maintained in this state during 12 hours and up to 3 days to ensure proper clathration. The cell is maintained at a pressure and temperature above the estimated clathrate stability curve until about $140 \mathrm{~K}$, where the gas is evacuated while pursuing the temperature descent to its minimum. Infrared spectra are therefore recorded in the $7000-650 \mathrm{~cm}^{-1}$ range, raising the temperature step by step from its minimum. The recording of each spectrum typically lasts one hour and the temperature is raised at $1 \mathrm{~K}$ to $2 \mathrm{~K} / \mathrm{min}$ between each temperature step.

\section{Results}

We have measured the NIR to Mid-IR $\mathrm{CH}_{4}, \mathrm{CO}, \mathrm{CO}_{2}$ and $\mathrm{H}_{2} \mathrm{~S}$ clathrate spectra. The cage dependent vibrational modes shifts has been identified using mixtures with other molecules. From a fundamental aspect, such clathrate hydrate spectra provide fingerprints and templates to test the validity of molecular potentials used by theoreticians to describe the ice behavior. This research 
focused on the clathrate hydrate spectroscopy topic, and discussions about the comparisons with astrophysical and planetary spectra are detailed in the bibliographic references related to this work.

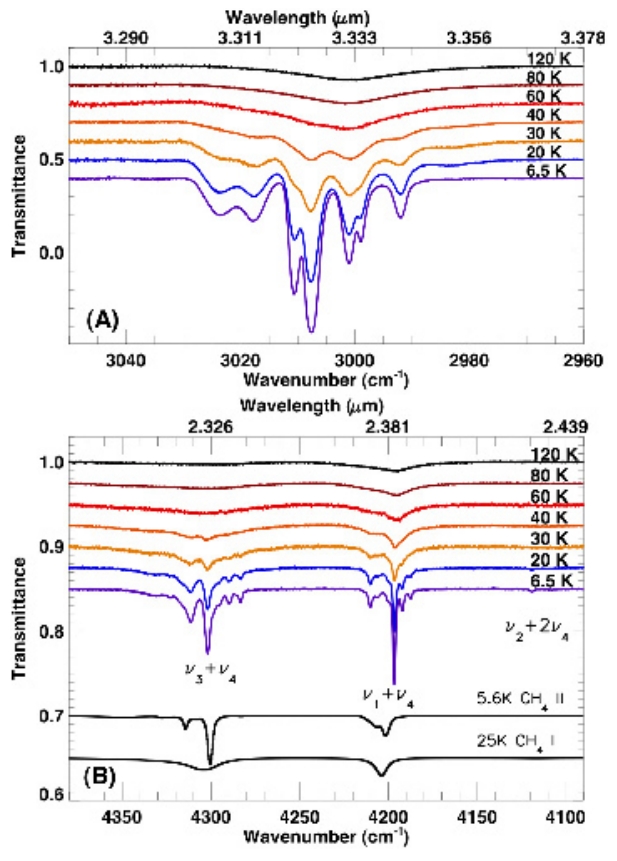

Fig. 1. Temperature-dependent spectra of methane clathrate hydrate in the $\mathrm{CH}$ stretching mode (A) and in the first combination modes (B) regions. A pure $\mathrm{CH} 4$ ice spectrum is shown for comparison, in its two phases below $20.9 \mathrm{~K}$ (cubic phase II) and just above (phase I). Spectra are shifted vertically for clarity.

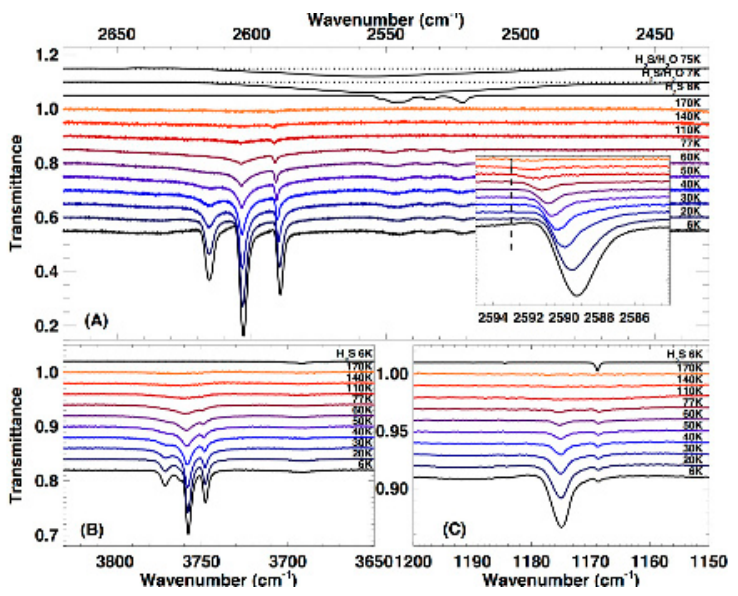

Fig. 2. Temperature-dependent infrared spectra of the hydrogen sulfide clathrate hydrate in the $\mathrm{SH}$ stretching mode $(\mathrm{A})$, combinations $(\mathrm{B})$, and bending $(\mathrm{C})$ regions. In (A), two transitions are observed in the 77 to $170 \mathrm{~K}$ temperature range, pertaining to the type I small and large cages environments. Below $77 \mathrm{~K}$, a third component appears.

Astrophysical clathrate hydrates must be searched for by Spectroscopy. If the present spectra can be used to identify their possible presence in astrophysical bodies, they also provide a spectral dictionary to follow, in the laboratory, their kinetics incorporation into ice. Kinetics is overlooked for their formation (especially for cold objects : interstellar grains, comets, TNOs) and must take an increasing importance experimentally. Many other clathrate hydrates are under spectroscopic investigation.

\section{References}

1. Dartois, E., Duret, P., Marboeuf, U., Schmitt, B., Icarus 220, 427-434 (2012)

2. Dartois E., Icarus 212(2): 950-956 (2011)

3. Dartois E, Deboffle D, Bouzit M., Astron. Astrophys. 514: id.A49 (2010)

4. Dartois E, Schmitt B., Astron. Astrophys. 2009;504:869-873 (2009)

5. Dartois E, Deboffle D., Astron. Astrophys. 490:L19-L22 (2008) 